\title{
Artificial Intelligence Is around Us. Let's Pick Up!
}

\author{
Jaehwa Cho \\ Division of Pulmonary and Critical Care Medicine, Department of Internal Medicine, Gangnam Severance Hospital, Yonsei University College of Medicine, \\ Seoul, Korea
}

We watched the exciting scene in which Lee Se Dol played against AlphaGo and were thrilled to win one game. This case shows that artificial intelligence (AI) is around us. AI has drawn attention to how it will change human life in the future.

The deep learning technology that is based on AI is applied to the medical field. It is especially advanced in the field of imaging like radiology. In April 2018, U.S. Food and Drug Administration (FDA) permitted marketing of the first medical device to use AI to detect diabetic retinopathy in adults who have diabetes [1]. Also, U.S. FDA permitted marketing of imagen OsteoDetect that was $\mathrm{AI}$ algorithm for aiding providers in detecting wrist fractures in adult patients [2]. FDA is working to lay down new rules and regulations that would enable regulators to keep up with new technologies such as AI and encourage innovation. In Korea, last year the Korean Ministry of Food and Drug Safety (MFDS) made a new regulatory guideline for approval and examination of AI-based and big data-based medical devices (software). In May 2018, the first AI-based medical device, VUNO's Vunomed-BoneAge, gained government approval in Korea. This device determine the bone age by studying X-ray images [3]. In August, MFDS approved two additional AI-based medical devices_Lunit's Lunit Insight and JLK Inspection's JBS-01K. The Lunit Insight analyzes a patient's X-ray and displays the degree of the suspected pulmonary nodule, which in turn helps doctors diagnose pulmonary nodule. JLK's JBS-01K helps doctors to make an appropriate treatment option for patients with cerebral infarction by providing them with cerebral infarct pattern using the patient's magnetic resonance imaging [4]. There are many start up business ventures for AI and big databased technologies.

The AI-based software, IBM Watson for Oncology has been introduced and used for practice. Recently, STAT pressed Watson supercomputer often spit out erroneous cancer treatment advice and the company medical specialists and customers identified "multiple examples of unsafe and incorrect treatment recommendations [5]." As a new drug developed after clinical trials encountered previously unrecognized adverse events, AI-based software meets unheard of problems, errors. The experts for AI and big data argued the most important thing is appropriate, highly qualified, and sufficient data to train the AI software.

In the field of critical care, there are many signals such as vital signs, electrocardiogram, airway pressure, and flow waves on mechanical ventilators. We hear a lot of alarm sounds from intensive care unit monitoring and devices. It is hard to identify what is real alarm sound. I think AI-based devices would help us to make it clear and solid. There are numerous items and ideas to improve public and patient's safety. The doctors should provide nice ideas and data for training AI-based devices. Now we have to be the game maker not a bystander.

\section{Editorial}

Received: August 22, 2018

Accepted: August 22, 2018

Corresponding author

Jaehwa Cho

Division of Pulmonary and Critical

Care Medicine, Department of Internal Medicine, Gangnam Severance Hospital, Yonsei University College of Medicine, 211 Eonju-ro, Gangnam-gu, Seoul 06273, Korea Tel: +82-2-2019-3317

Fax: +82-2-3463-3883

E-mail: jhcho66@yuhs.ac

Copyright @ 2018 The Korean Society of Critical Care Medicine

This is an Open Access article distributed under the terms of Creative Attributions Non-Commercial License (http:// creativecommons.org/li-censes/by-nc/4.0/) which permits unrestricted noncommercia use, distribution, and reproduction in any medium, provided the original work is properly cited. 


\section{CONFLICT OF INTEREST}

No potential conflict of interest relevant to this article was reported.

\section{ORCID}

Jaehwa Cho

https://orcid.org/0000-0002-3432-3997

\section{REFERENCES}

1. U.S. Food and Drug Administration. FDA permits marketing of artificial intelligence-based device to detect certain diabetes-related eye problems [Internet]. Silver Spring (MD): U.S. Food and Drug Administration; 2018 [cited 2018 Aug 23]. Available from: https://www.fda.gov/newsevents/newsroom/pressannouncements/ucm604357.htm.

2. U.S. Food and Drug Administration. FDA permits marketing of artificial intelligence algorithm for aiding providers in detecting wrist fractures [Internet]. Silver Spring (MD): U.S. Food and Drug Administration; 2018 [cited 2018 Aug 23]. Available from: https://www.fda.gov/newsevents/newsroom/pressannouncements/ucm608833.htm.

3. Ministry of Food and Drug Safety. First approval of AI-based medical device developed in Korea [Internet]. Cheongju: Ministry of Food and Drug Safety; 2018 [cited 2018 Aug 23]. Available from: http://www.mfds.go.kr/brd/m_99/view.do?seq=41992.

4. Ministry of Food and Drug Safety. Approval of two AI-based medical devices in Korea [Internet]. Cheongju: Ministry of Food and Drug Safety; 2018 [cited 2018 Aug 23]. Available from: http://www.mfds.go.kr/brd/m_99/view.do?seq=42985.

5. STAT. IBM's Watson supercomputer recommended 'unsafe and incorrect' cancer treatments, internal documents show [Internet]. Boston (MA): STAT; 2018 [cited 2018 Aug 23]. Available from https://www.statnews.com/2018/07/25/ibm-watson-recommended-unsafe-incorrect-treatments/. 